analyses the lowest category of $25(\mathrm{OH}) \mathrm{D}$ concentration was defined as reference. Heterogeneity among studies was evaluated by using the Cochran $\mathrm{Q}$ test and quantified by using the $\mathrm{I}^{2}$ statistical parameter. Stratified analyses were conducted to assess modification effects by selected factors. Publication bias was examined by using Funnel plot and Egger's regression asymmetry test. Random-effects meta-analyses were conducted using the metafor package in $\mathrm{R}$

Results Of 260 identified articles, 25 were included in the present review. Comparing the highest vs. the lowest category of prenatal $25(\mathrm{OH}) \mathrm{D}$ levels, the pooled beta coefficients were $0.95(95 \%$ CI $-0.03,1.93 ; \mathrm{p}=0.05)$ for cognition, and 0.88 (95\% CI $-0.18,1.93 ; \mathrm{p}=0.10)$ for psychomotor development. The pooled relative risk for ADHD was 0.72 (95\% CI, 0.59, $0.89 ; \mathrm{p}=0.002)$, and the pooled odds ratio for autism-related traits was $0.42(95 \% \mathrm{CI}, 0.25,0.71 ; \mathrm{p}=0.001)$. Stratified analyses showed stronger associations between $25(\mathrm{OH}) \mathrm{D}$ concentrations in early-mid pregnancy and cognition (beta coefficient 1.18, 95\% CI -0.16, 2.51; p=0.08), psychomotor performance (beta coefficient 1.43, 95\% CI $-0.65,3.52$; $\mathrm{p}=0.18$ ), and risk of autism-related traits (OR 0.28, 95\% CI $0.19,0.42 ; \mathrm{p}<0.01)$. There was little evidence for protective effects of high prenatal $25(\mathrm{OH}) \mathrm{D}$ for language development and behavior difficulties.

Conclusion This meta-analysis provides supporting evidence that increased prenatal exposure to $25(\mathrm{OH}) \mathrm{D}$ levels is associated with improved cognitive development and reduced risk of ADHD and autism-related traits later in life. Associations represent a potentially high public health burden given the current prevalence of vitamin D deficiency and insufficiency among childbearing aging and pregnant women.

\section{OP77 METHODS TO INCREASE RESPONSE RATES TO A POPULATION-BASED MATERNITY SURVEY: A COMPARISON OF TWO PILOT STUDIES}

S Harrison, J Henderson, F Alderdice, MA Quigley*. National Perinatal Epidemiology Unit, University of Oxford, Oxford, UK

\subsection{6/jech-2019-SSMabstracts.80}

Background Surveys are established methods for collecting population data that are unavailable from other sources; however, response rates to surveys are declining. This decline is exemplified in the National Maternity Surveys which are carried out in England at regular intervals by the National Perinatal Epidemiology Unit; the response rate declined from 63\% in 2006 to $29 \%$ in 2016. A number of methods have been identified to increase survey returns yet response rates continue to fall. We evaluated the impact of five selected methods on the response rate to pilot surveys, conducted prior to the 2018 National Maternity Survey.

Methods The pilot National Maternity Surveys were cross-sectional population-based questionnaire surveys of women who were three months postpartum selected at random from birth registrations. Women received a postal questionnaire, which they could complete on paper, online or verbally over the telephone. An initial pilot survey was conducted (pilot 1, $\mathrm{n}=1,000$ ) to which the response rate was lower than expected. Therefore, a further pilot survey was conducted (pilot 2, $\mathrm{n}=2,000$ ) using additional selected methods with the specific aim of increasing the response rate. The additional selected methods used for all women in pilot 2 were: pre- notification, a shorter questionnaire, more personable survey materials, an additional reminder, and inclusion of quick response (QR) codes to enable faster access to the online version of the survey. To assess the impact of the selected methods, response rates to pilot surveys 1 and 2 were compared.

Results The response rate increased significantly from $28.7 \%$ in pilot 1 to $33.1 \%$ in pilot $2(+4.4 \%, 95 \% \mathrm{CI}: 0.88-7.83$, $\mathrm{p}=0.02$ ). Analysis of weekly returns according to time from initial and reminder mail-outs suggests that this increase was largely due to the additional reminder. Most respondents completed the paper questionnaire rather than taking part online or over the telephone in both pilot surveys. However, the overall response to the online questionnaire almost doubled from $1.8 \%$ in pilot 1 to $3.5 \%$ in pilot 2 , corresponding to an absolute difference of $1.7 \%$ (95\%CI:0.45-2.81, p=0.01), suggesting that $\mathrm{QR}$ codes might have facilitated online participation.

Conclusion Declining survey response rates may be ameliorated with the use of selected methods. Further studies should evaluate the effectiveness of each of these methods using randomised controlled trials and identify novel strategies for engaging populations in survey research.

\section{Methodological Issues}

\section{OP78 A CAUSAL INFERENCE PERSPECTIVE ON COMPOSITIONAL DATA AND COLLIDER 'BIAS'}

1,2 KF Arnold*, 1,2 L Berrie, 1,2,3PWG Tennant, 1,2,3 MS Gilthorpe. 'Leeds Institute for Data Analytics, University of Leeds, Leeds, UK; ${ }^{2}$ School of Medicine, University of Leeds, Leeds, UK; ${ }^{3}$ The Alan Turing Institute, London, UK

\subsection{6/jech-2019-SSMabstracts.81}

Background Compositional data (CD) comprise the parts of some whole, for which all parts sum to that whole; the whole may vary across individual units of analysis or remain fixed. Such data are common in many contexts, where interest often lies in understanding the effect of a particular part in relation to a subsequent outcome. Many of the inherent challenges associated with analysing $\mathrm{CD}$ have been discussed previously, though not within a formal causal framework by utilising directed acyclic graphs (DAGs). We use DAGs to consider the specific issue of collider bias as it pertains to CD.

Methods We demonstrate how to depict CD using DAGs, and identify two distinct effect estimands in the generic case: (1) the 'unbiased' (total) effect, and (2) the 'collider biased' effect. We consider each effect in the context of three specific example scenarios involving CD with variable or fixed totals: (1) the relationship between the economically active population and area-level gross domestic product (GDP) (variable total); (2) the relationship between fat consumption and body weight (variable total); and (3) the relationship between time spent sedentary and body weight (fixed total). For each scenario, we consider the distinct interpretation of each effect, and the resulting implications for related analyses.

Results In scenario (1), the 'unbiased' effect represents the average change in GDP that results from adding economically active individuals to the area whilst doing nothing to the population of economically inactive individuals, whereas the 'collider biased' effect represents the average change that results from swapping economically inactive individuals for economically active ones. In scenario (2), the 'unbiased' effect 